Bayarmaa Mandzhieva, MD

Senior Resident, Internal Medicine

Residency, AdventHealth, Orlando, FL
Akriti Jain, MD

Senior Resident, Internal Medicine

Residency, AdventHealth, Orlando, FL
Manoucher Manoucheri, MD, FACP

Associate Program Director, Internal Medicine

Residency, AdventHealth, Orlando, FL

\title{
Median arcuate ligament syndrome: Incidental finding or real problem?
}
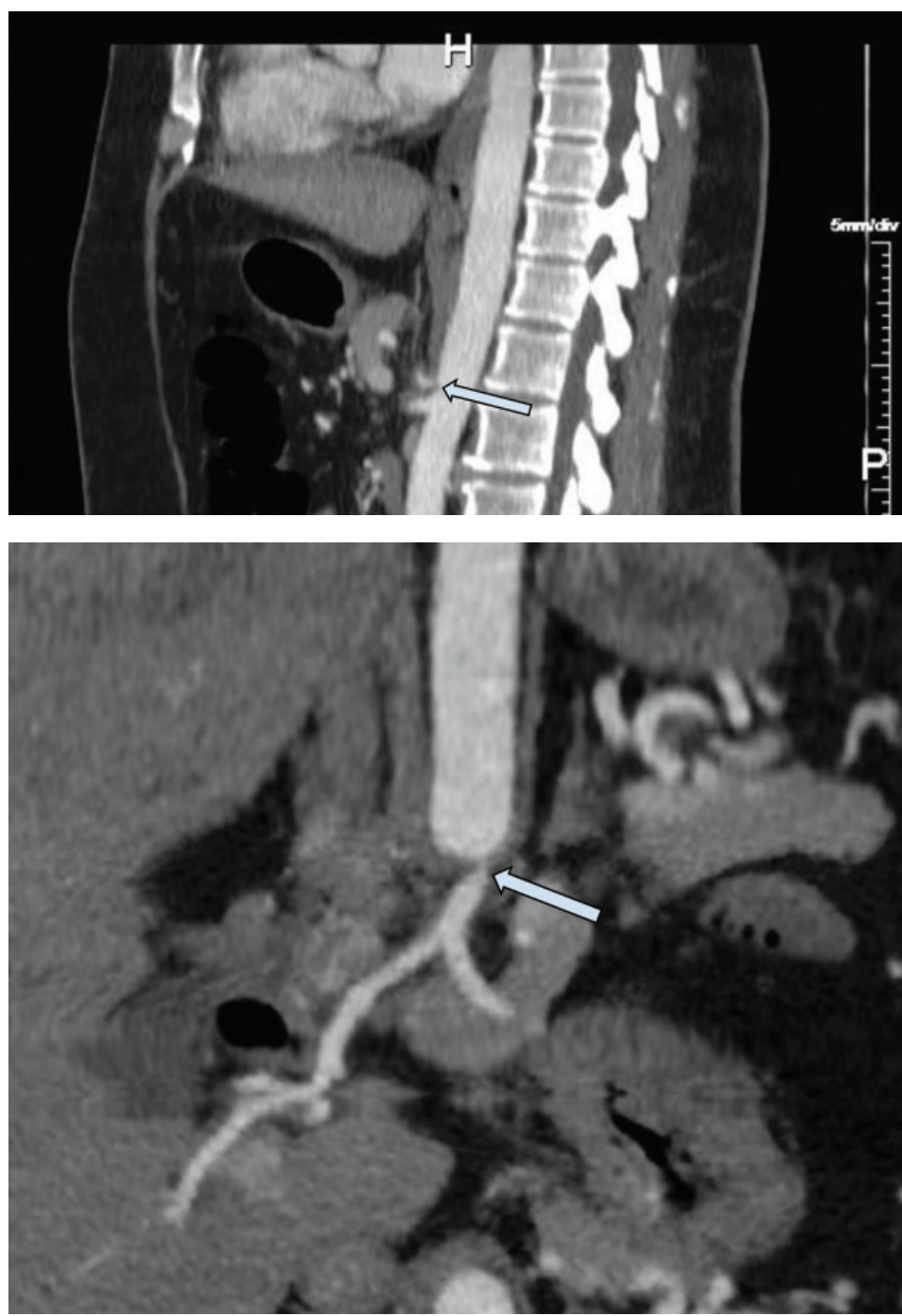

Figure 1. Contrast-enhanced computed tomographic angiography, sagittal (top) and 3-D coronal (bottom) views, showed severe stenosis at the origin of the celiac artery (arrow) with associated soft-tissue attenuation, suggestive of median arcuate ligament syndrome.

doi:10.3949/ccjm.88a.20052
43-Year-old woman was admitted with A pain in the epigastrium and right upper quadrant that radiated to the back and was associated with nausea and dry heaves. The pain had started suddenly 5 days previously. She described it as burning, pressure-like, and intermittent without any relation to meals. She had no vomiting, fevers, chills, change in bowel habits, or unintended recent weight loss. She had a history of chronic gastroesophageal reflux disease and had multiple episodes of this epigastric pain over the past few years, but she always thought it was related to her reflux, and it was never this severe. She had never undergone upper endoscopy.

See related editorial, page 143

On physical examination, she had mild tenderness to palpation in the epigastric area. Laboratory studies were essentially normal.

Right upper quadrant ultrasonography showed an unremarkable gallbladder and biliary tree. Computed tomography (CT) of the abdomen revealed compression of the celiac artery by the arcuate ligament. She subsequently underwent CT angiography of the abdomen, which showed severe stenosis of the origin of the celiac artery with associated soft-tissue attenuation, suggestive of median arcuate ligament syndrome (MALS) (Figure 1). The celiac artery beyond the area of narrowing was widely patent. There were prominent arterial collaterals in the peripancreatic region, with some prominence of the gastroduodenal artery likely related to contribution to the celiac distribution from the superior mesenteric artery (Figure 2). The superior mesenteric artery and inferior mesenteric artery were widely patent with no radiographic evidence of bowel ischemia. 
It was not clear if her symptoms were related to artery compression or to a severe form of gastritis or peptic ulcer disease, with MALS as an incidental finding. She was subsequently evaluated by a gastroenterologist and a general surgeon for possible laparoscopic release of the ligament.

The patient's symptoms were not typical for MALS; they were new in onset and she had no weight loss, no abdominal pain after eating, and no food aversion. The surgeon did not attribute her abdominal pain to MALS and did not recommend surgery.

The gastroenterologist recommended upper endoscopy, which showed no acute pathology to explain her symptoms, and biopsy studies were negative.

Her symptoms improved during her hospital stay, and she was counseled to follow up with her primary care physician for further testing if required.

\section{THE CLINICAL PICTURE OF MALS}

The median arcuate ligament is a fibrous arch connecting the crura of the diaphragm forming the aortic hiatus and lying superior to the celiac artery. MALS, also known as Dunbar syndrome or celiac artery compression syndrome, is a rare phenomenon caused by extrinsic compression of the celiac trunk by the median arcuate ligament.

Women with MALS outnumber men by $2: 1$ to $3: 1$, and the typical age of onset is in the fourth and fifth decades. ${ }^{1}$ Often, history and physical findings are nonspecific. The most common clinical manifestation is chronic epigastric abdominal pain, most of the time postprandial or exercise-induced. Other symptoms include nausea, emesis, bloating, weight loss, and fear of the pain triggered by eating, leading to food avoidance. Physical examination may reveal epigastric tenderness or a bruit that is amplified with expiration, but these are nonspecific.

\section{What causes the epigastric pain?}

Theories regarding the pathophysiology of epigastric pain associated with MALS include foregut ischemia due to compressed celiac artery, midgut ischemia due to vascular steal syndrome, and overstimulation of the celiac plexus with subsequent splanchnic vasoconstriction and ischemia. Recently, ideas about

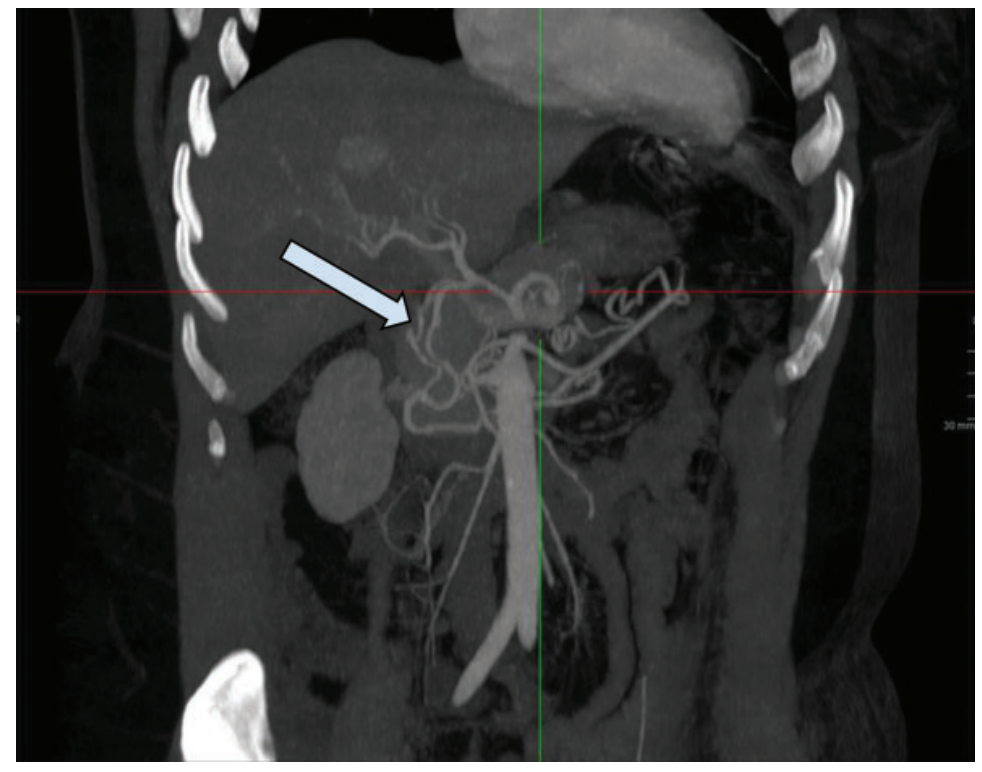

Figure 2. Contrast-enhanced computed tomographic angiography showed prominent poststenotic arterial collaterals in the peripancreatic region (arrow), with prominence of the gastroduodenal artery related to contribution to the celiac distribution from the superior mesenteric artery, which indicates the stenosis is chronic and hemodynamically significant.

the etiology of MALS have shifted from its being a vascular disease to a neurogenic disorder The patient's with compression of the surrounding celiac plexus and ganglion. ${ }^{2}$

\section{Mimics of MALS}

MALS resembles several other abdominal disorders in its symptoms, posing a diagnostic challenge for the clinician. It can be mistaken for gastroparesis, gastritis, peptic ulcer disease, hepatitis, cholecystitis, biliary dyskinesia, appendicitis, chronic pancreatitis, colorectal malignancy, or chronic mesenteric ischemia secondary to atherosclerotic disease. Most patients undergo an extensive workup for other diagnoses with abdominal ultrasonography, abdominal CT, upper endoscopy, and hepatobiliary iminodiacetic acid scanning.

MALS is considered a diagnosis of exclusion, and it can coexist with other intraabdominal pathologies and be a confounding factor.

CT angiography, magnetic resonance angiography, and duplex abdominal ultrasonography during inspiration and deep expiration are the most common diagnostic studies for 
MALS. The increasing use of CT in the assessment of abdominal pain has led to more frequent diagnosis of MALS.

Also, many patients who have no symptoms exhibit radiographic evidence of celiac compression, and mild compression can normally be seen during expiratory-phase CT angiography; inspiratory imaging can confirm that the narrowing is real. Petnys et $\mathrm{al}^{3}$ showed that $3 \%$ of patients without symptoms have celiac artery compression on CT angiography. In a retrospective study, Heo et $\mathrm{al}^{4}$ showed that $87 \%$ of patients with MALS had no symptoms, and the condition was incidentally diagnosed by CT. Anatomically, up to $24 \%$ of the population may have compression of the celiac artery; however, fewer than $1 \%$ of them have symptoms. ${ }^{5}$

To enhance the benefit of surgical intervention, studies aimed at improving the ability to reliably diagnose MALS are required. Surgery should be reserved for patients who would benefit from it, and patient selection continues to be challenging, as there is relatively poor correlation between the radiographic findings of celiac artery compression and the presence or severity of symptoms. It is generally accepted that asymptomatic or incidentally discovered MALS does not warrant intervention.

Laparoscopic release of the arcuate ligament has become a widely accepted treatment. Endovascular therapy may be necessary as well, given the possible recurrence of stenosis. Multidisciplinary assessment by a general surgeon, vascular surgeon, radiologist, and gastroenterologist is helpful.

Cienfuegos et $\mathrm{al}^{6}$ offered the following selection criteria for laparoscopic treatment: young woman, intense postprandial pain, greater than $70 \%$ stenosis of the trunk, and development of collateral circulation.

\section{DISCLOSURES}

The authors report no relevant financial relationships which, in the context of their contributions, could be perceived as a potential conflict of interest.

\section{REFERENCES}

1. Sidawy AN, Perler BA. Rutherford's Vascular Surgery and Endovascular Therapy. 9th ed. Philadelphia, PA: Elsevier; 2018.

2. Weber JM, Boules $\mathbf{M}$, Fong $\mathbf{K}$, et al. Median arcuate ligament syndrome is not a vascular disease. Ann Vasc Surg 2016; 30:22-27. doi:10.1016/j.avsg.2015.07.013

3. Petnys A, Puech-Leão P, Zerati AE, et al. Prevalence of signs of celiac axis compression by the median arcuate ligament on computed tomography angiography in asymptomatic patients. J Vasc Surg 2018; 68(6):1782-1787. doi:10.1016/j.jvs.2018.04.044

4. Heo S, Kim HJ, Kim B, Lee JH, Kim J, Kim JK. Clinical impact of collat- eral circulation in patients with median arcuate ligament syndrome. Diagn Interv Radiol 2018; 24(4):181-186. doi:10.5152/dir.2018.17514

5. Horton KM, Talamini MA, Fishman EK. Median arcuate ligament syndrome: evaluation with CT angiography. Radiographics 2005; 25(5):1177-1182. doi:10.1148/rg.255055001

6. Cienfuegos JA, Estevez MG, Ruiz-Canela M, et al. Laparoscopic treatment of median arcuate ligament syndrome: analysis of longterm outcomes and predictive factors. J Gastrointest Surg 2018; 22(4):713-721. doi:10.1007/s11605-017-3635-3

Address: Bayarmaa Mandzhieva, MD, AdventHealth, 2501 N Orange Avenue, Suite 235, Orlando, FL 32804;

bayarmaa.mandzhieva.md@adventhealth.com 